\section{NMDA Receptor Encephalitis with Concurrent Autoimmune Hypophysitis Presented with Acute Psychosis}

Chawit Lopimpisuth, M.D. ${ }^{1}$, Phuksuphang Thippawan, M.D. ${ }^{1}$, Rudruidee Karnchanasorn, M.D. ${ }^{2}$

${ }^{\mathrm{l}}$ Mahidol University, Faculty of Medicine Siriraj Hospital, Bangkok, Thailand

${ }^{2}$ University of Kansas Medical Center, Division of Metabolism,

Endocrinology and Genetics, Kansas City, KS

Received May 28, 2020; Accepted for publication.July 27, 2020; Published online Oct. 20, 2020 https: doi.org 10.17161 kijm.voll3.14764

\section{INTRODUCTION}

Acute psychosis is a very challenging diagnosis. There can be a wide range of etiologies, from psychiatric problems to autoimmune encephalitis, toxin, medication, and metabolic disorder. ${ }^{1}$ Autoimmune encephalitis, such as N-methyl-D-aspartate (NMDA) receptor encephalitis, is one of the prominent organic causes of acute psychosis, with the prevalence of $0.6 / 100,000{ }^{2}$ Hypophysitis is also a rare disease. The annual incidence of hypophysitis is estimated to be 1 in 7 - 9 million, ${ }^{3}$ but recently, due to immunotherapy treatment, the incidence is significantly rising. ${ }^{4}$ We report the unusual case of a female patient with a recent diagnosis of hypophysitis with secondary adrenal insufficiency on steroid-replacement therapy who presented with acute psychosis with a presumed diagnosis of steroid-induced psychosis. Subsequent brain magnetic resonance imaging (MRI) confirmed the diagnosis of limbic encephalitis with hypophysitis.

\section{CASE REPORT}

A 41-year-old female presented to the Emergency Department after multiple days of agitation, confusion, and insomnia. She had a history of thrombotic thrombocytopenic purpura with complete remission following plasma exchange therapy and high dose steroid up to $60 \mathrm{mg} /$ day of prednisone and lymphocytic hypophysitis with recent diagnosis of secondary adrenal insufficiency and hypothyroidism. Her husband noted that she repeated several times that she is the "Righteousness of Christ". She denied having visual changes or headache. There was no reported history of substances used and no past psychiatric problems. She was taking hydrocortisone $20 \mathrm{mg}$ daily and levothyroxine $75 \mathrm{mcg}$ daily, but her husband reported self-increased dose of the hydrocortisone to $30 \mathrm{mg}$ daily and levothyroxine $100 \mathrm{mcg}$ daily in the last three days due to fatigue.

The patient was admitted to the medical service for presumed steroid induced psychosis. Vital signs were within normal limits. Neurological exam was significant for agitation and confusion. The patient was oriented to person only but retained ability to follow basic commands. Babinski response was absent bilaterally. Hyperreflexia was found in both lower and upper extremities. Laboratory studies and urinalysis were unrevealing of any profound metabolic disturbance except for low thyroid stimulating hormone. Computed tomography (CT) of the brain revealed no intracranial or neurovascular lesions. A concern for steroid induced psychosis was noted, although unlikely since the reported hydrocortisone dose was not significantly high. However, hydrocortisone dose was reduced to $15 \mathrm{mg}$ /day without clinical improvement.

\section{KANSAS JOURNAL of MEDICINE}

Two days following admission, Acyclovir was given due to possibility of herpes encephalitis. An electroencephalogram (EEG) showed structural lesion involving the left temporal area. No epileptiform activity was found. MRI of the brain showed redevelopment of lymphocytic hypophysitis and development of ill-defined fluid-attenuated inversion recovery (FLAIR) hyperintensities and expansion in the region of the left amygdala favored for autoimmune limbic encephalitis. A lumbar puncture revealed a cerebrospinal fluid (CSF) profile of 32 white blood cells, 1 red blood cells, $89 \%$ lymphocytes, protein of 62 , glucose of 50 , and oligoclonal bands were detectable. Tests for herpes simplex virus (HSV), human herpesvirus 6, cryptosporidium, and fungal infection were negative. Gram stain and culture were negative. Acyclovir was discontinued when polymerase chain reaction for HSV was unremarkable.

An evaluation for paraneoplastic syndrome was initiated. Tumor markers and CSF autoimmune antibody serologies were sent, and the patient underwent CT scans of the chest, abdomen, and pelvis for any occult malignancy. All were unremarkable. The patient was started on methylprednisolone but failed to respond. She subsequently was given intravenous immunoglobulin (IVIG) for presumed antiNMDA receptor encephalitis with minimal but gradual improvement in neurologic status. NMDA receptor antibody was positive, which confirmed the final diagnosis of anti-NMDA receptor encephalitis concurrent with recurrent hypophysitis.

\section{DISCUSSION}

Limbic encephalitis (LE) is characterized by rapidly progressive short-term memory loss, psychiatric symptoms, and seizures. LE can have varied presentation and delayed diagnosis is common. Diagnosis is based on clinical, imaging study, and presence of antibody. ${ }^{5}$

The most well-known antibody is NMDA receptor antibody. Typically, symptoms emerge in multiple stages. Patients usually develop virus-like prodromal symptoms, with headache, lethargy, and fever, followed by progressive behavioral changes, memory deficits, confusion, and psychosis within two weeks. ${ }^{5,6}$ The majority of cases are related to paraneoplastic syndrome, especially teratoma, which is found in up to one third of adult patients. Other tumors that have been described in other literatures, including neuroblastoma, Hodgkin lymphoma, tumors of the breast, thymus, and lung, are rare. ${ }^{7}$ However, recognizing the limbic encephalitis is often difficult for the clinicians, due to multiple lists of other differential diagnosis, such as, brain metastases, toxicity of treatments, and other metabolic complications.

Steroid induced psychosis is a rare but devastating side effect of glucocorticoid therapy. The prevalence rate of these disorders among patients that received corticosteroid therapy is varied from $1.8 \%$ to $57 \%$ of patients. ${ }^{8}$ The incidence rate of psychiatric disorders is correlated directly to dose and time of glucocorticoids exposure with variable presentations. Rate of psychiatric disorders occurs in $1.3 \%$ of cases when the dose is less than $40 \mathrm{mg}$ daily, and reaches $18.4 \%$ for 


\section{KANSAS JOURNAL of MEDICINE}

ORGANIC CAUSES OFACUTE PSYCHOSIS

continued.

doses of $80 \mathrm{mg}$ daily. ${ }^{9}$ Usually symptoms happen in the first week, with median time to onset of 11.5 days, with $39 \%$ of disturbances occurring during the first week, $62 \%$ within two weeks, and $83 \%$ within six weeks of treatment initiation. ${ }^{10}$

Hypophysitis, inflammation of the pituitary gland, leads to hypopituitarism and compression of sella and parasella structures and is likely an autoimmune disease. It usually presents with headache, visual disturbance, and adrenal insufficiency. ${ }^{11}$

Our patient met the diagnostic criteria of NMDA receptor encephalitis with concurrent diagnosis of hypophysitis. This was the first case report in literature of both diagnoses in a patient who presented with acute psychosis. The initial diagnosis of steroid-induced psychosis was unlikely due to low dose of hydrocortisone. When patients present with acute psychosis for the first time, a wide range of differential diagnosis should be made to screen for potential organic causes.

\section{REFERENCES}

${ }^{1}$ Keshavan MS, Kaneko Y. Secondary psychoses: An update. World Psychiatry 2013; 12(1):4-15. PMID: 23471787.

2 Dubey D, Pittock SJ, Kelly CR, et al. Autoimmune encephalitis epidemiology and a comparison to infectious encephalitis. Ann Neurol 2018; 83(1):166-177. PMID: 29293273.

${ }^{3}$ Faje A. Hypophysitis: Evaluation and management. Clin Diabetes Endocrinol 2016; 2:15. PMID: 28702249.

${ }^{4}$ Faje A. Immunotherapy and hypophysitis: Clinical presentation, treatment, and biologic insights. Pituitary 2016; 19(1):82-92. PMID: 26186958.

${ }^{5}$ Jagtap SA, Das GK, Kambale HJ, Radhakrishnan A, Nair MD. Limbic encephalitis: Clinical spectrum and long-term outcome from a developing country perspective. Ann Indian Acad Neurol 2014; 17(2):161-165. PMID: 25024565.

${ }^{6}$ Hermetter C, Fazekas F, Hochmeister S. Systematic review: Syndromes, early diagnosis, and treatment in autoimmune encephalitis. Front Neurol 2018; 9:706. PMID: 30233481.

7 Newman MP, Blum S, Wong RC, et al. Autoimmune encephalitis. Intern Med J 2016; 46(2):148-157. PMID: 26899887.

${ }^{8}$ Wada K, Yamada N, Sato T, et al. Corticosteroid-induced psychotic and mood disorders: Diagnosis defined by DSM-IV and clinical pictures. Psychosomatics 2001; 42(6):461-466. PMID: 11815680.

9 Warrington TP, Bostwick JM. Psychiatric adverse effects of corticosteroids. Mayo Clin Proc 2006; 81(10):1361-1367. PMID: 17036562.

${ }_{10}$ Drug-induced convulsions. Report from Boston Collaborative Drug Surveillance Program. Lancet 1972; 2(7779):677-679. PMID: 4115818.

${ }^{11}$ Prete A, Salvatori R. Hypophysitis. In: Endotext [Internet]. South Dartmouth (MA): MDText.com, Inc. August 15, 2018. PMID: 30160871.

Keywords: anti-NMDA receptor encephalitis, autoimmune hypophysitis, psychosis 